\title{
Nonperturbative Approach to the Description of Molecular Excitation by Ultrashort Laser Pulses
}

\author{
A.A. Biryukov a and M.A. Shleenkov \\ Samara State University, 1 Akademik Pavlov Str. Samara 443011, Russia
}

\begin{abstract}
We study the problem of excitation of molecules by an ultrashort laser pulse sequence by the use of path-integral approach in energy states space. Using numerical calculations of path-integral we evaluate laser field parameters at which there are quantum resonances in rotational dynamics of dinitrogen isotopes. So we describe recent experimental results [Phys. Rev. Lett. 109, 043003 (2012)] theoretically.
\end{abstract}

Keywords: path integral, rotational excitation of molecules.

The modern development of laser radiation technologies induces theoretical and experimental investigations of the dynamics of quantum objects (such as atoms or molecules) under the action of intense electromagnetic field of different forms.

This dynamics is principally non-linear, because the probability is high of multiphoton processes (absorption and emission more the one photon) and nonresonant processes (electromagnetic field frequency is far from quantum transitions frequency). We note the recent studies of different rare gases multiphoton ionization [1--3], of multiphoton photoemission of the $\mathrm{Au}(111)$ surface state with 800-nm laser pulses [4], of multiphoton transitions in $\mathrm{GaSb} / \mathrm{GaAs}$ quantum-dot intermediate-band solar cells [5], of three-photon electromagnetically induced absorption in a ladder-type atomic system [6].

There are certain difficulties for theoretical studies of these processes and for simulations of quantum objects dynamics that interact with laser field. Thus, different approximations are used. For example, there are two- or three-level quantum system models [7] and rotating wave approximation [8]. For high-intensity laser field the perturbation theory runs into problems. It is necessary to calculate the large number of terms. High-order perturbation theory for miltilevel quantum system dynamics [9] was considered.

The perspective approach to theoretical studies of this quantum processes is path integral (functional integral) formalism, which are formulated by R.P. Feynman [10, 11] and based on P.A.M. Dirac ideas $[12,13]$. At present this formalism is an abundantly used approach in many fields of physics: lattice theories in QCD simulations [14] and those in graphene [15], semiclassical approachs in atom optics [16, 17], influence functional approach in quantum theory $[18,19]$ and many others.

In this paper we present new method of calculating the transition probability of a quantum system interacting with electromagnetic field by the path integral formalism. We construct the amplitude and probability of quantum transition as path integrals in energy states space. The algorithm of path

\footnotetext{
${ }^{\mathrm{a}}$ Corresponding author: biryukov@samsu.ru
} 
integral calculation was developed. This approach enables us to perform computer simulations of molecule dynamics induced by a laser field.

By the deduced formulas we describe quantum resonances in dynamics of nitrogen molecules, that interact with a sequence of ultrashort laser pulses. The obtained results are in good agreement with the experimental data [20].

The approach developed is appliable to nonperturbative studies of different multiphoton and nonresonant processes.

The work is supported by the Ministry of Education and Science of Russian Federation (grant 2.870.2011).

\section{References}

1. N. Gerken, S. Klumpp, A. A. Sorokin et al Phys. Rev. Lett. 112, 213002 (2014)

2. R. Guichard, M. Richter, Rost et al J. Phys. B: At. Mol. Opt. Phys. 46, 164025 (2013)

3. M. Richter, M. Y. Amusia, Bobashev et al, Phys. Rev. Lett. 102, 163002 (2014)

4. F. Sirotti, N. Beaulieu, A. Bendounan et al Phys. Rev. B 90, 035401 (2014)

5. J. Hwang, K. Lee, A. Teran et al Phys. Rev. App. 1, 051003 (2014)

6. H. S. Moon, and T. Jeong, Phys. Rev. A 89, 033822 (2014)

7. S. Cho, H. Moon, Y. Chough, et al Phys. Rev. A 89, 053814 (2014)

8. J. Spiegelberg and E. Sjqvist, Phys. Rev. A 88, 054301 (2013)

9. A. A Biryukov and B. V. Danilyuk, Proc. SPIE 7024, 702405 (2008)

10. R. P. Feynman, Rev. Mod. Phys. 20, 367 (1948)

11. R. P. Feynman and A. R. Hibbs, Quantum Mechanics and Path Integrals (McGraw-Hill Companies, 1965).

12. P. A. M. Dirac, Physikalische Zeitschrift der Sowjetunion 3, 64-72 (1933)

13. P. A. M. Dirac, Principles of Quantum Mechanics (Oxford University Press 1982 (fourth edition))

14. V. Bornyakov, E.-M. Ilgenfritz, Martemyanov et al Phys. Rev. D 87, 114508 (2013)

15. S. N. Valgushev, E. V. Luschevskaya, Pavlovsky, et al JETP Lett. 98, 445 (2013)

16. A. B. Bichkov, A. A. Mityureva and V. V. Smirnov, Phys. Rev. A 79, 013402 (2009)

17. A. B. Bichkov, A. A. Mityureva and V. V. Smirnov, J. Phys. B: At. Mol. Opt. Phys. 44, 135601 (2011)

18. R. P. Feynman and F. L. Vernon, J., Annals of Physics 24, 118 (1963)

19. A. Biryukov and M. Shleenkov, PoS QFTHEP 2013 076, 1 (2013)

20. S. Zhdanovich, C. Bloomquist, Floss, et al Phys. Rev. Lett. 109, 043003 (2012) 